\title{
Time-dependent search for neutrino emission from Mrk 421 and Mrk 501 observed by the HAWC gamma-ray observatory
}

\author{
Mukharbek Organokov \\ IPHC - Institut Pluridisciplinaire Hubert Curien - Universite de Strasbourg et CNRS/IN2P3, \\ 23 rue du Loess, BP 28, 67037 Strasbourg Cedex 2, France \\ E-mail: mukharbek.organokov@iphc.cnrs.fr
}

\section{Agustin Sanchez-Loza*}

INFN - Sezione di Bari, Via Edoardo Orabona 4, 70125 Bari, Italy

E-mail: agustin.sanchez@ba.infn.it

\section{Thierry Pradier}

IPHC - Institut Pluridisciplinaire Hubert Curien - Universite de Strasbourg et CNRS/IN2P3,

23 rue du Loess, BP 28, 67037 Strasbourg Cedex 2, France

E-mail: thierry.pradiereiphc.cnrs.fr

\section{on behalf of the ANTARES Collaboration}

The principles of a search for high energy neutrino emission in coincidence with very high energy (VHE; 0.1-100 TeV) gamma-ray flares from two bright extragalactic sources, based on the data collected in 2012-2016 by the ANTARES neutrino detector and High Altitude Water Cherenkov (HAWC) gamma-ray observatory, are presented. The ANTARES telescope observes with high duty cycle an instantaneous field of view of $2 \pi$. To study variable sources like blazars, it is crucial to achieve unbiased monitoring and an instrument like HAWC is capable of long term and continuous monitoring of the source with nearly $100 \%$ duty cycle. Markarian 421 (Mrk 421) and Markarian 501 (Mrk 501) are the brightest and the closest BL Lac objects known. In contrast to other types of active galactic nuclei (AGN), BL Lacs are characterized by rapid and largeamplitude flux variability. Such radio-loud AGNs with collimated jets aligned to the line of sight, are candidate sources of the observed high energy cosmic rays and of accompanying neutrinos and gamma rays produced in hadronic interactions with the surrounding medium.

35th International Cosmic Ray Conference - ICRC2017

10-20 July, 2017

Bexco, Busan, Korea

${ }^{*}$ Speaker. 


\section{Introduction}

The VHE extragalactic sky is dominated by emission from blazars [1], a class of non-thermal jet-powered AGN known as radio-loud AGN. The spectral energy distribution (SED) of blazars can be described with two components: one low-energy from radio to X-ray and one high-energy from $\mathrm{X}$-ray to $\mathrm{TeV}$. The low energy component is commonly assumed to come from synchroton emision of accelerated emissed. The origin of the high-energy part is still under discussion: either from inverse compton in the leptonic processes or imbeded protons [2]. Some hadronic scenarios introduce relativistic protons to explain the high-energy bump generally seen in the $\mathrm{MeV}$ to $\mathrm{TeV}$ range for BL Lac objects. In the hadronic framework, this component attributed to either proton-synchrotron emission or radiation from secondary products of $\mathrm{p} \gamma$ or pp interactions [2, 3, 4]. Acceleration of protons is assumed to occur by the first-order diffusive Fermi mechanism at the shock, resulting in a generic $\mathrm{E}^{-2}$ differential spectrum. Energy loss processes which occur during acceleration include $\mathrm{p} \gamma \rightarrow \mathrm{N} \pi$ and $\mathrm{p} \gamma \rightarrow \mathrm{p}+\mathrm{e}^{+} \mathrm{e}^{-}$in the dense radiation fields and pp collisions in the gas. All three processes contribute to an energetic electromagnetic component, either through $\pi^{0} \rightarrow \gamma \gamma$ or by production of electrons. Both photo-meson production and pp collisions also give rise to neutrinos via $\pi^{ \pm} \rightarrow \mu^{ \pm} v_{\mu} \bar{v}_{\mu} \rightarrow \mathrm{e}^{ \pm} v_{\mathrm{e}} \bar{v}_{\mathrm{e}} v_{\mu} \bar{v}_{\mu}$ decay chain [3]

This analysis focuses on the search of spatial/temporal correlation between neutrinos detected by ANTARES and $\gamma$-ray emission from flares detected by HAWC from Mrk 421 and Mrk 501, the first and the second extragalactic object discovered in the TeV energy band. Moreover, these are two of four sources detected in the $0.6-2.0 \mathrm{TeV}$ band by Fermi-LAT out of the Galactic plane, $|\mathrm{b}|$ $>5^{\circ}$, and at low redshift, $\mathrm{z}<0.3$ [5]. Both sources have been significantly detected in the 2HAWC catalog [6], and flare information were recently reported in [7] (see section 3.1).

Mrk 421 and Mrk 501 are the brightest and closest BL Lac objects known, at luminosity distances $\mathrm{d}_{\mathrm{L}}=134 \mathrm{Mpc}$ with redshift $\mathrm{z}=0.031$ and $\mathrm{d}_{\mathrm{L}}=143 \mathrm{Mpc}$ with redshift $\mathrm{z}=0.033$ respectively. Both are classified as high-peaked BL Lac objects (HBL). Mrk 421 is known to exhibit a high degree of variability in its emission and yearly average fluxes are known to vary between a few tenths and 1.9 times the flux of the Crab Nebula. Variability of Mrk 421 has been observed down to time scales of hours or less and its spectral shape is known to vary with its brightness. Various studies of Mrk 501 at $\mathrm{TeV}$ energies have shown different features of low flux states emission and extreme outbursts [7]. The VHE spectrum of Mrk 421 has been successfully modeled with both leptonic and hadronic models and conclusive results have been achieved yet about origin and both models are still under debate [8]. As the nearest blazars to Earth, both are excellent sources to test the blazar-neutrino connection scenario, especially during flares where time-dependent neutrino searches may have a higher detection probability.

The IceCube collaboration has performed a time integrated search for the most significant a priori source candidates selected on the basis of $\gamma$-rays observations or astrophysical modeling that predicts neutrino emission [9]. No significant excess was found leading to an differential flux limit of $\mathrm{dN} / \mathrm{dE} \leq 11.71 \times 10^{-12} \mathrm{TeV}^{-1} \mathrm{~cm}^{-2} \mathrm{~s}^{-1}$ for Mrk 421 and $\mathrm{dN} / \mathrm{dE} \leq 8.11 \times$ $10^{-12} \mathrm{TeV}^{-1} \mathrm{~cm}^{-2} \mathrm{~s}^{-1}$ for Mrk 501. Additional searches in IceCube [10] show no correlated neutrinos.

Similar time-dependent analyses have been performed in the past using ANTARES data, in particular for flaring blazars [11]. 


\section{The ANTARES neutrino telescope}

The ANTARES Neutrino Telescope (Astronomy with a Neutrino Telescope and Abyss environmental RESearch) is a Cherenkov detector located in the Mediterranean sea, $40 \mathrm{~km}$ off the coast of Toulon, France $\left(42^{\circ} 48^{\prime} \mathrm{N}^{\circ} 10^{\prime}\right)$, at a depth of 2,475 meters. Currently, ANTARES is the largest neutrino telescope in the Northern Hemisphere and it is designed primarily to search for $\mathrm{E}>100 \mathrm{GeV}$ muons resulting from the charged-current $(\mathrm{CC})$ interactions of $v_{\mu}$ in the vicinity of the detector. The median angular resolution for $\mathrm{E}^{-2}$ for tracks is $\sim 0.5^{\circ}$ [12]. The search of such astrophysical neutrinos origin has become a key mission in high-energy astrophysics after the discovery from IceCube of a cosmic neutrino flux of still unknown origin [13].

\subsection{Data selection}

The data set covers the period from the January $1^{\text {st }}, 2012$ to December $31^{\text {st }}, 2016$ (MJD: 55927-57753) leading to effective detector livetime of 1209 days, covering the same period of observation as HAWC. The search relies on track-like event signatures, so only CC interactions of muon neutrinos considered. Atmospheric neutrinos are the main source of background. An additional source of background is due to the mis-reconstructed atmospheric muons. The muon track reconstruction returns two quality parameters, namely the track-fit quality parameter, $\Lambda$, and the estimated angular uncertainty on the fitted muon track direction, $\beta$. Cuts on these parameters are used to improve the signal-to-noise ratio. $\Lambda$ wil be optimised source by source. To ensure a good directional reconstruction of the selected $v$ candidates, $\beta<1$ is required [14].

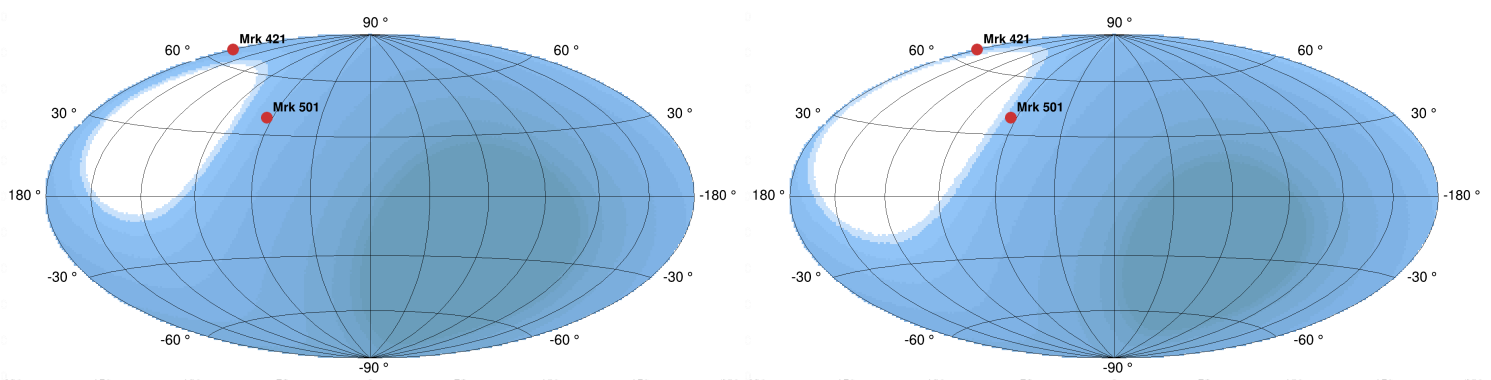

Figure 1: ANTARES visibility of the sky ranging from 0 (white) to $100 \%$ (dark blue) with $10 \%$ step. Left: Upgoing and slightly downgoing (angle above the horizon below $8.62^{\circ}$ ) for $\cos (\theta)>-0.15$; Right: Only Upgoing $\cos (\theta)>0$.

Due to the fact that Mrk 421 and Mrk 501 are located at the edge of ANTARES visibility (see Fig. 1) and sources are below the horizon only $\sim 6$ hours per day, a small amount of down-going events can be accepted to gain $\sim 15 \%$ in visibility (see Fig. 1), and increase an observation time (see Fig. 2). This is done by application of the cut on cosine of zenith angle of the events. In this analysis events with $\cos (\theta)>-0.15$ are selected.

\section{The HAWC gamma-ray telescope}

The HAWC Observatory is located at an elevation of 4,100 $\mathrm{m}$ above sea level on the flanks of the Sierra Negra volcano in the state of Puebla, Mexico $\left(18^{\circ} 99^{\prime} \mathrm{N}, 97^{\circ} 18^{\prime} \mathrm{W}\right)$. The detector 


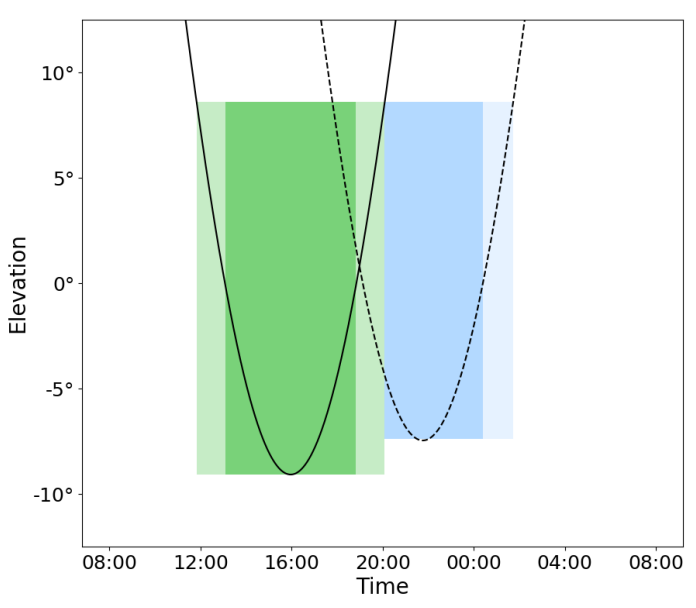

Figure 2: Source elevation as a function of time for Mrk 421 (solid) and for Mrk 501 (dash) at the ANTARES position for January $1^{\text {st }}, 2016$. Corresponding color boxes represent the elevation ranges (height) and source observation time by ANTARES (width) for $\cos (\theta)>0$ (dark) and $\cos (\theta)>-0.15$ (light).

array installation began in February 2012; HAWC-300 fully operational began operation on March 20, 2015. Covering an area of $22,000 \mathrm{~m}^{2}$, the array consists of 300 water Cherenkov detectors (WCDs), with 190,000 liters each and instrumented with 4 PMTs to detect Cherenkov light from charged particles in extensive air showers. The design of HAWC is optimized for the detection of air showers induced by $\gamma$-rays in 0.1-100 TeV. With field of view $\sim 2$ steradians HAWC can monitor any source over $2 / 3$ of the sky for up to 6 hours per day and most sensitive to sources between declinations $-26^{\circ}$ and $+64^{\circ}[7,15,16]$. Such capabilities make unprecedented $\mathrm{TeV}$ light curve data available for studying flaring behavior of blazars. In addition, scanning a large part of the sky with such sensitivity increase the chances to find bright flare events from established and new extragalactic sources [7].

\subsection{Light curves of blazars}

HAWC has made clear detections of Mrk 421 and Mrk 501. Preliminary blazar light curves from data taken in 2013-2014 with the partial HAWC array were shown in [16]. In this analysis HAWC-300 data of first long-term TeV light curve studies with single-transit intervals are used, collected over 17 months between November 26 th 2014 and April 20 th 2016 [7].

In [7] the maximum-likelihood approach was used to test if the daily flux measurements in a light curve are consistent with a source flux that is constant in time over the whole period under consideration. The best-fit flux value $F_{i}$ for time interval $i$ is fitted from the minimisation of the following likelihood function [7]:

$$
\mathrm{dN} / \mathrm{dE}=\mathrm{F}_{\mathrm{i}}(\mathrm{E} /(1 \mathrm{TeV}))^{-\gamma} \exp \left(-\mathrm{E} / \mathrm{E}_{\mathrm{cut}}\right)
$$

converted to Crab Units by dividing by the HAWC measurement of the average Crab Nebula $\gamma$-ray flux $1.89 \times 10^{-11} \mathrm{ph} \mathrm{cm}^{-2} \mathrm{~s}^{-1}$ above $1 \mathrm{TeV}$ in order to allow comparisons between the sources. The main characteristics of the gamma-ray flux for the two sources are summarised in Table 1. 
Table 1: Some parameters from [7]

\begin{tabular}{ccc}
\hline \hline & Mrk 421 & Mrk 501 \\
\hline Highest daily flux* & $26.94 \pm 3.7$ & $16.7 \pm 2.3$ \\
Average flux* & $4.53 \pm 0.14$ & $1.74 \pm 0.08$ \\
$\Gamma^{* *}$ & $2.21 \pm 0.14_{\text {stat }} \pm 0.20_{\text {sys }}$ & $1.60 \pm 0.30_{\text {stat }} \pm 0.20_{\text {sys }}$ \\
$\mathrm{E}_{\text {cut }}^{* *}$ & $\mathrm{E}_{0}=5.4 \pm 1.1_{\text {stat }} \pm 1.0_{\text {sys }}$ & $\mathrm{E}_{0}=5.7 \pm 1.6_{\text {stat }} \pm 1.0_{\text {sys }}$ \\
\hline
\end{tabular}

* in $10^{-12} \mathrm{ph} \mathrm{cm}^{-2} \mathrm{~s}^{-1}$ units; fluxes calculated above $2 \mathrm{TeV}$ for Mrk 421 and above $3 \mathrm{TeV}$ for Mrk 501.

${ }^{* *}$ Spectral index and exponential cut-off, derived from spectral fit.

If a light curve is variable, the Bayesian blocks algorithm can be used to find an optimal data segmentation into regions that are well represented by a constant flux, within the statistical uncertainties. Bayesian blocks algorithm is used to identify distinct flux states (see Fig. 3), both Mrk 421 and Mrk 501 show clear variability on time scales of one day. Taking advantage of gamma-ray flux variation time information from potential neutrino emitters as in Fig. 3, significantly reduce the $v$ background and improve the signal-to-noise discrimination.
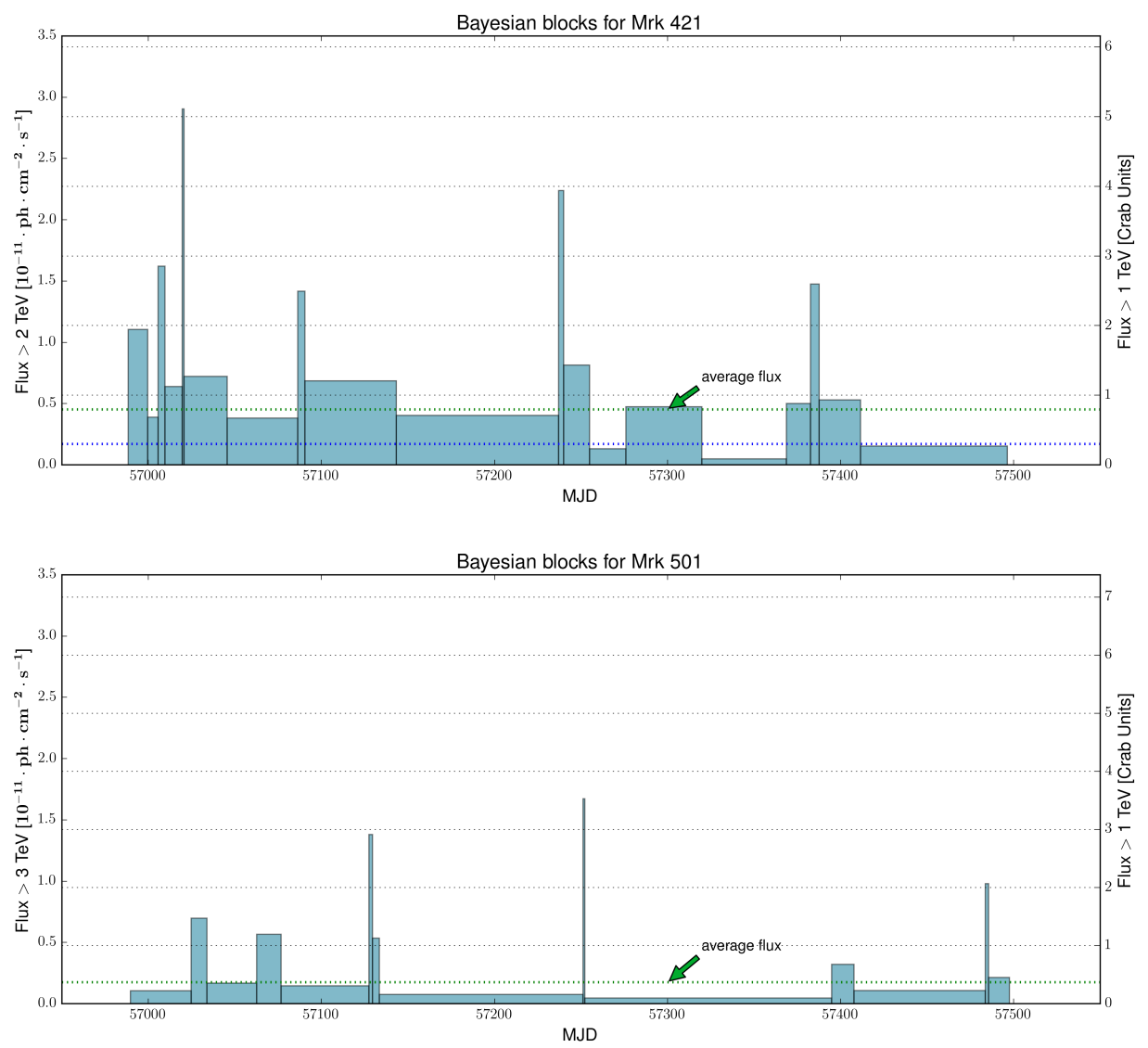

Figure 3: Flux light curve for Mrk 421 (top) and Mrk 501 (bottom) for 17 months; the green dotted lines represent the average fluxes, $\sim 0.8 \mathrm{CU}$ and $\sim 0.3 \mathrm{CU}$ respectively. The value for Mrk 421 is higher than previous estimates for an upper limit to the baseline flux $\sim 0.3 \mathrm{CU}$ (blue). Adapted from [7] 


\section{Time-dependent search method}

A time-dependent search for neutrino candidades in coincidence with $\gamma$-rays from astrophysical sources is performed using an unbinned likelihood-ratio maximization method [11]. This is done via the test statistics, $\lambda$, defined as the ratio of the probability for the hypothesis of the background and signal $\left(\mathrm{H}_{\mathrm{sig}+\mathrm{bkg}}\right)$, called $\mathrm{H}_{1}$, over the probability of only background $\left(\mathrm{H}_{\mathrm{bkg}}\right)$, called $\mathrm{H}_{0}$ :

$$
\lambda=\sum_{\mathrm{i}=1}^{\mathrm{N}} \ln \frac{\mathrm{P}\left(\mathrm{x}_{i} \mid \mathrm{H}_{\mathrm{sig}+\mathrm{bkg}}\left(\mathrm{N}_{\mathrm{S}}\right)\right)}{\mathrm{P}\left(\mathrm{x}_{i} \mid \mathrm{H}_{\mathrm{bkg}}\right)},
$$

where $\mathrm{N}_{\mathrm{S}}$ (unknown) and $\mathrm{N}$ (known) are expected number of signal events and total number of events in the considered data sample, $\mathrm{x}_{i}$ are observed event properties $\left(\delta_{\mathrm{i}}, \mathrm{RA}_{\mathrm{i}}, \mathrm{dE} / \mathrm{dX}_{i}\right.$, and $\left.\mathrm{t}_{i}\right)$.

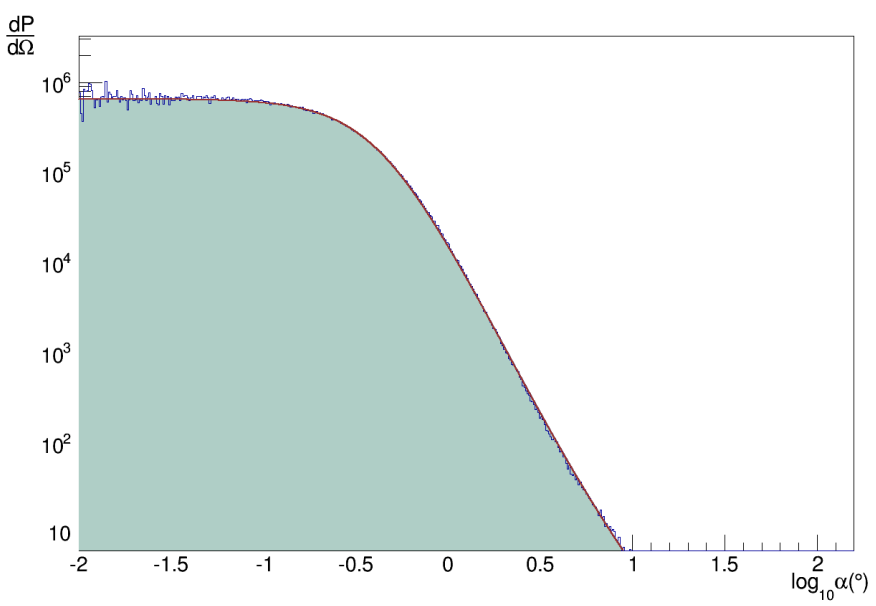

Figure 4: Sample of PSF parametrization for Mrk 421. The PSF parametrisation before normalisation (red line) with $\log _{10} \alpha$ distribution (shaded rea) for $\mathrm{E}^{-2}$ spectrum and $\Lambda_{\text {cut }}>-5.2$.

To perform the analysis, the ANTARES data sample is parametrized as two-component mixture of signal and background. The signal is expected to be small so that the full data direction scrambled can be used as an estimation of background. $S_{i}$ and $B_{i}$ are defined as the probability density functions (PDF) respectively for signal and background for an event $i$, at time $t_{i}$, energy, declination $\delta_{\mathrm{i}}$. The logarithm of the likelihood $\mathrm{L}$ is:

$$
\ln (\mathrm{L})=\left(\sum_{\mathrm{i}=1}^{\mathrm{N}} \ln \left[\mathrm{N}_{\mathrm{S}} \mathrm{S}_{\mathrm{i}}+\mathrm{N}_{\mathrm{B}} \mathrm{B}_{\mathrm{i}}\right]\right)-\left[\mathrm{N}_{\mathrm{S}}+\mathrm{N}_{\mathrm{B}}\right]
$$

To distinguish the signal-like events from the background ones, for each event three types of PDFs are used, based on: direction $\mathrm{P}_{\mathrm{s}}\left(\alpha_{\mathrm{i}}\right)$ and $\mathrm{P}_{\mathrm{b}}\left(\delta_{\mathrm{i}}\right)$ for signal and background part respectively, where parameter $\alpha_{i}$ represents the angular distance between the direction of the event $i$ and direction to the source, and $\delta_{i}$ is the declination of the event $\mathrm{i}$; energy $\mathrm{P}_{\mathrm{s}}(\mathrm{E})$ and $\mathrm{P}_{\mathrm{b}}(\mathrm{E})$; time $\mathrm{P}_{\mathrm{s}}(\mathrm{t}+\operatorname{lag})$ and $\mathrm{P}_{\mathrm{b}}(\mathrm{t})$. These ingredients are determined using the ANTARES Monte Carlo simulations (MC) and data (see section 2.1). The background PDFs are all computed using data only. The shape of the time PDF for the signal event is extracted directly from the $\gamma$-ray light curve assuming a proportionality 
between the $\gamma$-ray and the neutrino fluxes. A possible lag up to \pm 5 days has been introduced in the likelihood. The energy PDF for the signal event is produced according to the studied energy spectrum. One of the most important parameter in point source search is the angular distance to the source, characterized by the Point Spread Function (PSF) - $\mathrm{P}_{\mathrm{s}}\left(\alpha_{\mathrm{i}}\right)$ (see Fig. 4), which is defined as the probability density of $\alpha$ per unit solid angle, $\Omega$ :

$$
\mathrm{P}_{\mathrm{S}}\left(\alpha_{\mathrm{i}}\right)=\frac{\mathrm{dP}}{\mathrm{d} \Omega}=\frac{\mathrm{d} \alpha_{\mathrm{i}}}{\mathrm{d} \Omega} \frac{\mathrm{dP}}{\mathrm{d} \alpha_{\mathrm{i}}}=\frac{1}{2 \pi \sin \alpha_{\mathrm{i}}} \frac{\mathrm{dP}}{\mathrm{d} \alpha_{\mathrm{i}}},
$$

where $\alpha_{i}=\left|\alpha_{i}^{\text {true }}-\alpha_{i}^{\text {rec }}\right|$ is the difference between simulated neutrino direction $\alpha_{i}^{\text {true }}$ and reconstructed muon direction $\alpha_{i}^{\text {rec }}$.

Combining all terms considered before, the final likelihood is:

$$
\ln (\mathrm{L})=\left(\sum_{\mathrm{i}=1}^{\mathrm{N}} \ln \left[\mathrm{N}_{\mathrm{S}} \cdot \mathrm{P}_{\mathrm{S}}(\alpha) \cdot \mathrm{P}_{\mathrm{S}}(\mathrm{E}) \cdot \mathrm{P}_{\mathrm{s}}(\mathrm{t}+\mathrm{lag})+\mathrm{N}_{\mathrm{B}} \cdot \mathrm{P}_{\mathrm{b}}(\sin (\delta)) \cdot \mathrm{P}_{\mathrm{b}}(\mathrm{E}) \cdot \mathrm{P}_{\mathrm{b}}(\mathrm{t})\right]\right)-\left[\mathrm{N}_{\mathrm{S}}+\mathrm{N}_{\mathrm{B}}\right]
$$

The test statistics is evaluated by generating pseudo-experiments (PEX) simulating background and signal around the considered source according to $\mathrm{H}_{0}$ and $\mathrm{H}_{1}$ hypothesis. The obtained value of $\lambda$ for the data is compared to the value obtained by PEX. The discovery potential is the average number of signal events required to achieve a p-value lower than $5.7 \cdot 10^{-7}$ for $5 \sigma$ discovery.

\section{Conclusion}

The HAWC detector operates nearly continuously and it is currently the most sensitive wide field-of-view telescope in the very promising VHE band $\sim 0.1$ and $\sim 100 \mathrm{TeV}$. Therefore, it opens prospects to study the most energetic astrophysical phenomena in the Universe as well as understand the mechanisms that power them and endeavour to break the mystery of their origin. Taking into account the flare timing information given by gamma-ray observations, even for long-duration flares, should improve the efficiency of the search for a neutrino counterpart with ANTARES. The next generation KM3NeT neutrino telescope [17], currently being built in the Mediterranean Sea, will provide more than an order of magnitude improvement in sensitivity [18]; therefore, such sources are promising candidates as high energy neutrino emitters for an improved future timedependent search.

\section{References}

[1] M. Cerruti et al. [HESS Collaboration], "Target of Opportunity observations of blazars with H.E.S.S.”, AIP Conf.Proc. 1792 (2017) no.1, 050029 [arXiv:1610.05523][astro-ph.HE]

[2] A. Zech, M. Cerruti, D. Mazin, "Expected signatures from hadronic emission processes in the TeV spectra of BL Lacertae objects", Astron.Astrophys. 602 (2017) A25 [arXiv:1703.05937][astro-ph.HE]

[3] T.K. Gaisser, F. Halzen, T. Stanev, "Particle Physics with High Energy Neutrinos", Phys. Rep. 258 (1995) 173 [arXiv:hep-ph/9410384]

[4] J.G. Learned, K. Mannheim, "High-energy neutrino astrophysics", Ann. Rev. Nucl. Part. Sci. 50 (2000) 679-749 
[5] M. Ackermann et al., ”2FHL: The Second Catalog of Hard Fermi-LAT Sources”, Astrophys. J., 222, 5. (2016) [arXiv:1508.04449][astro-ph.HE]

[6] A. Abeysekara et al. [HAWC Collaboration], ”The 2HWC HAWC Observatory Gamma Ray Catalog”, Astrophys.J. 843 (2017) no.1, 40 [arXiv:1702.02992][astro-ph.HE].

[7] A. Abeysekara et al. [HAWC Collaboration], "Daily monitoring of TeV gamma-ray emission from Mrk 421, Mrk 501, and the Crab Nebula with HAWC”, Astrophys.J., 841 (2017) no.2, 100 [arXiv:1703.06968][astro-ph.HE]

[8] A. Marinelli, B. Patricelli, N. Fraija, "Hadronic flares and associated neutrinos for Markarian 421", Published in IAU Symp. 313 (2015) 177-178 [arXiv:1505.03165][astro-ph.HE]

[9] Abbasi et al. [IceCube Collaboration], ”Time-Integrated Searches for Point-like Sources of Neutrinos with the 40-String IceCube Detector”, Astrophys J., 732 (2011), no.1, 18 [arXiv:1012.2137][astro-ph.HE]

[10] Aartsen et al. [IceCube Collaboration], "Searches for Extended and Point-like Neutrino Sources with Four Years of IceCube Data”, Astrophys.J., 796 (2014), no.2, 109 [arXiv:1406.6757][astro-ph.HE]

[11] A. Albert et al. [ANTARES Collaboration], ”Time-dependent search for neutrino emission from x-ray binaries with the ANTARES telescope”, JCAP 1704 (2017) no.04, 019 [arXiv:1609.07372][astro-ph.HE]

[12] A. Albert et al. [ANTARES collaboration], "New Constraints on all flavour Galactic diffuse neutrino emission with the ANTARES telescope" [arXiv:1705.00497][astro-ph.HE]

[13] Aartsen M G et al. [IceCube Collaboration], "Observation of High-Energy Astrophysical Neutrinos in Three Years of IceCube Data”, Phys. Rev. Lett. 113 (2014) 101101 [arXiv:1405.5303][astro-ph.HE]

[14] A. Albert et al. [ANTARES collaboration], ”First all-flavour Neutrino Point-like Source Search with the ANTARES Neutrino Telescope”, Submitted to: Phys.Rev.D [arXiv:1706.01857][astro-ph.HE]

[15] A. Abeysekara et al. [HAWC Collaboration], ”The HAWC Gamma-Ray Observatory: Design, Calibration, and Operation”, Proc. 33rd ICRC (2013) [arXiv:1310.0074][astro-ph.IM]

[16] R. J. Lauer and P. W. Younk [HAWC Collaboration], ”Results from monitoring TeV blazars with HAWC”, PoS ICRC2015 (2016) 716, [arXiv:1508.04479][astro-ph.HE]

[17] S. Adrian-Martinez et al. [KM3NeT Collaboration], ”Letter of intent for KM3NeT 2.0”, J. Phys. G43 no. 8, (2016) 084001 [arXiv:1601.07459][astro-ph.IM].

[18] A. Heijboer, on behalf of the ANTARES and KM3NeT Collaborations, "Highlights from ANTARES and KM3NeT", Highlight talk, these Proceedings. 